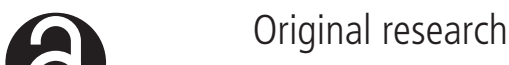

\section{Genomic temporal heterogeneity of circulating tumour DNA in unresectable metastatic colorectal cancer under first-line treatment}

\author{
Feng Wang, 1,2 You-Sheng Huang, 1,2,3 Hao-Xiang Wu, ${ }^{1,2}$ Zi-Xian Wang, 1,2 Ying Jin, 1,2 \\ Yi-Chen Yao, ${ }^{1,2}$ Yan-Xing Chen, ${ }^{1,2,3}$ Qi Zhao, ${ }^{1,2,3}$ Shifu Chen, ${ }^{4}$ Ming-Ming He, ${ }^{1,2}$ \\ Hui-Yan Luo, ${ }^{1,2}$ Miao-Zhen Qiu, ${ }^{1,2}$ De-shen Wang, ${ }^{1,2}$ Feng-Hua Wang, ${ }^{1,2}$ Mingyan Xu, ${ }^{4}$ \\ Yu-Hong Li, ${ }^{1,2}$ Rui-Hua Xu (D) ${ }^{1,2}$
}

Additional supplemental material is published online only. To view, please visit the journal online (http://dx.doi.org/ 10.1136/gutjnl-2021-324852)

For numbered affiliations see end of article.

\section{Correspondence to}

Professor Rui-Hua Xu and Dr Feng Wang, Department of Medical Oncology, Sun Yat-sen University Cancer Center, State Key Laboratory of Oncology in South China, Collaborative Innovation Center for Cancer Medicine, Sun Yatsen University, Guangzhou Guangdong, People's Republic of China; xurh@sysucc.org.cn, wangfeng@sysucc.org.cn

FW, Y-SH, H-XW, Z-XW and YJ are joint first authors.

Received 5 April 2021 Accepted 23 August 2021
Check for updates

(c) Author(s) (or their employer(s)) 2021. Re-use permitted under CC BY-NC. No commercial re-use. See rights and permissions. Published by BMJ.

To cite: Wang F, Huang Y-S, Wu H-X, et al. Gut Epub ahead of print: [please include Day Month Year]. doi:10.1136/

gutjn-2021-324852

\section{ABSTRACT}

Objective Circulating tumour DNA (ctDNA) sequencing is increasingly used in the clinical management of patients with colorectal cancer. However, the genomic heterogeneity in ctDNA during treatments and its impact on clinical outcomes remain largely unknown.

Design We conducted a prospective cohort study (NCT04228614) of 171 patients with unresectable metastatic colorectal cancer (mCRC) who underwent first-line treatment and prospectively collected blood samples with or without tumour samples from patients at baseline and sequentially until disease progression or last follow-up.

Results The RAS/BRAF alterations in paired baseline tissue and plasma samples from 63 patients displayed a favourable concordance $(81.0 \%, 51 / 63)$. After a period of first-line treatment (median time between baseline and last liquid biopsy, 4.67 months), 42.6\% (26/61) of RAS-mutant patients showed RAS clearance and $50.0 \%$ (5/10) of BRAF-mutant patients showed BRAF clearance, while $3.6 \%(3 / 84)$ and $0.7 \%$ (1/135) of patients showed new RAS or BRAF mutations in ctDNA. Patients with plasma RAS/BRAF clearance showed similar progressionfree survival (PFS) and overall survival (OS) with patients who remained RAS/BRAF wild-type, while much better outcomes than those who remained RAS/BRAF mutant. Patients who gained new RAS/BRAF mutations showed similar prognosis as those who maintained RAS/BRAF mutations, and shorter PFS and OS than those who remained RAS/BRAF wild-type.

Conclusion This prospective, serial and large-scale ctDNA profiling study reveals the temporal heterogeneity of $\mathrm{mCRC}$-related somatic variants, which should be given special attention in clinical practice, as evidenced by the finding that the shift in plasma RAS/BRAF mutational status can yield a drastic change in survival outcomes.

\section{INTRODUCTION}

Colorectal cancer (CRC) is the second leading cause of cancer-related death worldwide, and its incidence rates are increasing in many countries. ${ }^{12}$ Along with the progress of drug development, chemotherapy, targeted agents (ie, anti-epidermal growth factor receptor (EGFR) and anti-vascular endothelial growth factor (VEGF) therapy) and immune

\section{Significance of this study}

What is already known about this subject?

- The treatment strategies of metastatic colorectal cancer (mCRC) develop as the molecular diagnostics improve, and the failure of first and later lines of therapy may be caused by the molecular heterogeneity within patients over time.

- Plasma RAS mutation clearance in $\mathrm{mCRC}$ is increasingly used as a biomarker for selecting patients eligible for anti-EGFR rechallenge.

- Further exploration of the temporal heterogeneity of $\mathrm{mCRC}$-related somatic variants in circulating tumour DNA (ctDNA) is needed.

What are the new findings?

- After a period of first-line treatment, plasma RAS and BRAF clearance rates are $42.6 \%$ and $50.0 \%$, respectively, while RAS and BRAF acquisition rates are $3.6 \%$ and $0.7 \%$ in ctDNA.

- The shift in plasma RAS or BRAF mutational status correlates with the drastic change in survival outcomes.

- ERBB2 amplification, NTRK fusion and other actionable targets for clinical trials, including KRAS $^{G 12 C}$, BRAF ${ }^{\text {nonV600E }}$, PTEN, NF1, MTOR, MET, CDK12, CDKN2A, FGFR1/2/3, remain consistent over time in most patients.

How might it impact on clinical practice in the foreseeable future?

- This prospective, serial and large-scale ctDNA profiling study reveals the genomic temporal dynamics and heterogeneity of $\mathrm{mCRC}$ and provides solid evidence and insights to support the use of ctDNA sequencing in capturing the dynamic somatic mutational spectrum and predicting the prognosis of patients with $\mathrm{mCRC}$.

checkpoint inhibitors have largely reshaped the treatment of metastatic CRC (mCRC), which requires the precise stratification of patients according to their molecular features. ${ }^{3-5}$

In recent years, the rapid development of nextgeneration sequencing (NGS) technology has made 
it feasible for clinical application. The detection of actionable or prognostic somatic variants by NGS is important for guiding treatment decision-making for CRC. ${ }^{6}$ For instance, somatic RAS mutation is an indicator of primary resistance to anti-EGFR therapy and predicts poor survival outcomes. ${ }^{7}$ Somatic BRAF ${ }^{\mathrm{V} 600 \mathrm{E}}$ mutation is also a poor prognostic factor and the approved indication for triplet combination therapy (BRAF inhibitor, MEK inhibitor and anti-EGFR monoclonal antibody). ${ }^{8}$ Although the treatment strategies of mCRC develop as the molecular diagnostics improve, the resistance to first and later lines of therapy are caused by the molecular heterogeneity within patients over time. ${ }^{9} 10$

The main obstacles to answering these questions are the unfeasibility of repeated tissue biopsy and the spatial and temporal heterogeneity of tumour tissue. ${ }^{11} 12$ Liquid biopsy allows the examination of circulating tumour DNA (ctDNA), which is released into the bloodstream due to the breakdown of tumour cells. ${ }^{13}$ After much research, liquid biopsy can now be applied in clinical practice. ${ }^{14} 15$ The US Food and Drug Administration recently approved Guardant360 CDx, an NGS liquid biopsy panel, as the first liquid biopsy NGS companion diagnostic test for metastatic non-small-cell lung cancer. ${ }^{16}$ This marks a new era for mutation testing using liquid biopsy.

Emerging studies have shown that ctDNA analysis has the potential to be applied in the whole-course management of patients with CRC, including early diagnosis, minimal residual disease assessment, actionable target detection and treatment response monitoring in metastatic settings. ${ }^{14} 17$ For instance, we recently reported that ctDNA methylation profiles could be used in CRC screening. ${ }^{18}$ Several groups have also provided evidence supporting that ctDNA could reflect the existence of minimal residual disease postoperatively. ${ }^{19-21}$ Moreover, the serial ctDNA testing may help monitor treatment efficacy, with the early change in ctDNA serving as a marker of clinical response. ${ }^{22} 23$ And ctDNA could track RAS clones to monitor drug resistance or the potential to receive anti-EGFR rechallenge. ${ }^{24}$ However, studies on the evolution of somatic mutations of CRC in ctDNA under systemic therapy and its clinical significance are still lacking.

We conducted a prospective and observational study by enrolling patients with systemic therapy-naïve mCRC and employing serial ctDNA testing to monitor the temporal heterogeneity of somatic variants during first-line treatment and to investigate the potential correlations with clinical outcomes.

\section{RESULTS}

\section{Patient characteristics at baseline}

The study flow chart is presented in figure 1. In total, 171 patients with unresectable mCRC were enrolled. The clinical characteristics of the patients at baseline are listed in online supplemental table S1. Baseline RAS and BRAF ${ }^{\mathrm{V} 600 \mathrm{E}}$ mutations were detected in ctDNA from 74 (43.3\%) and 11 (6.4\%) patients, respectively. For first-line treatment, 94 (55.0\%) patients received chemotherapy plus bevacizumab, 51 (29.8\%) patients received chemotherapy only, $25(14.6 \%)$ patients received chemotherapy plus cetuximab and only $1(0.6 \%)$ patient with confirmed high microsatellite instability status received immunotherapy. A strong

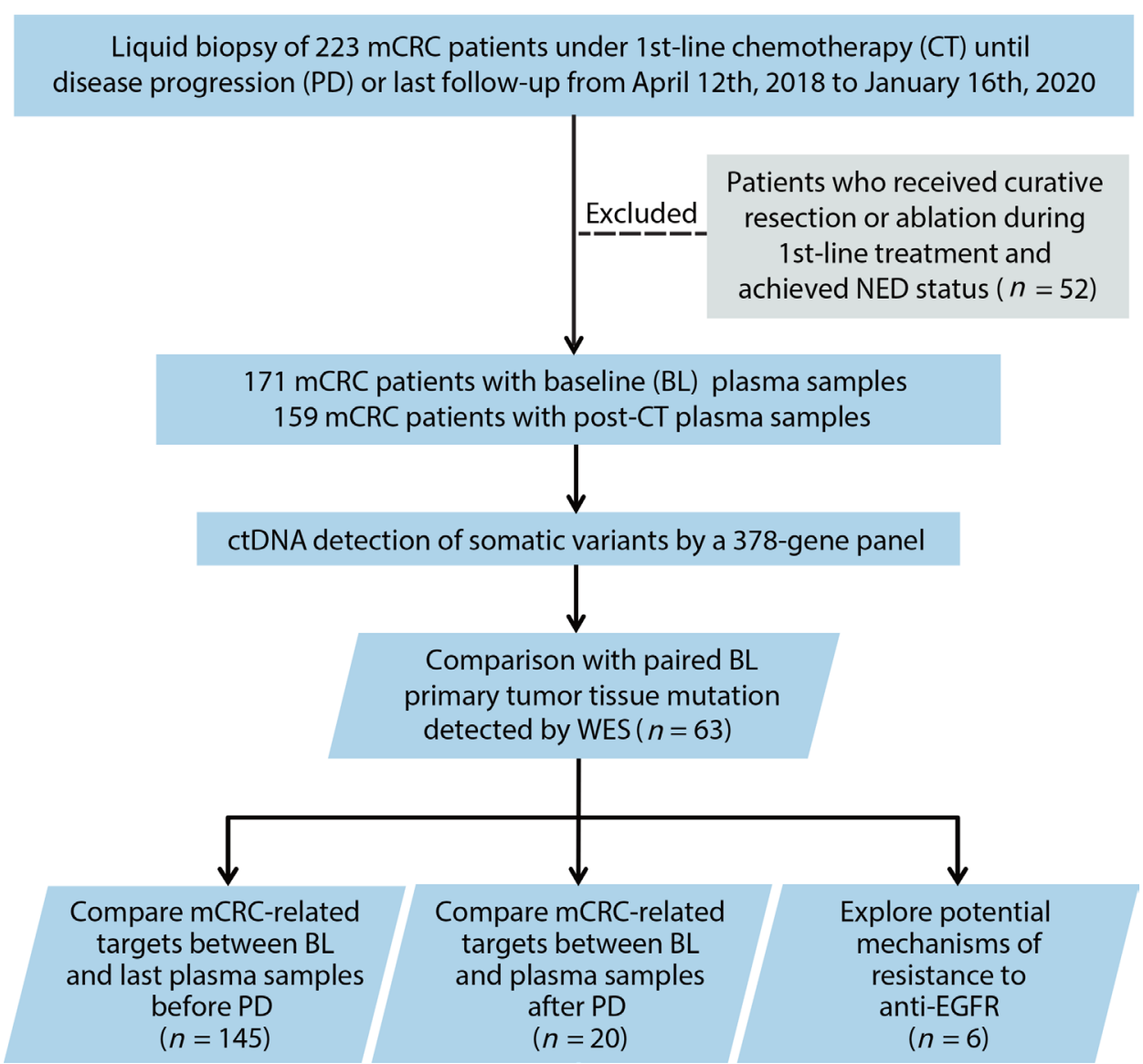

Figure 1 Flowchart of study design and patient selection. ctDNA, circulating tumour DNA; mCRC, metastatic colorectal cancer; NED, no evidence of disease; WES, whole-exome sequencing. 
A
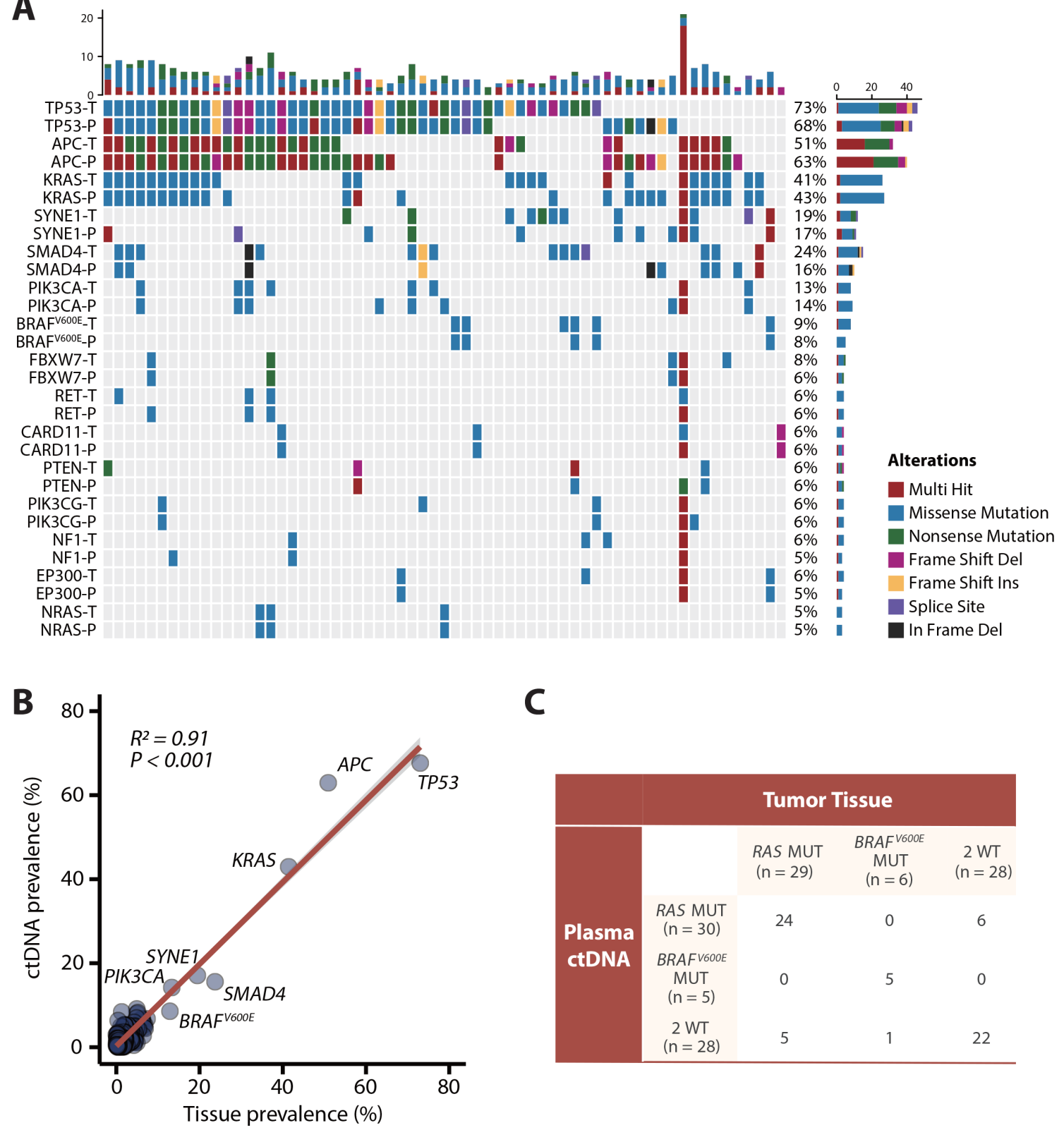

Figure 2 Concordance analysis of baseline mutations in paired plasma and tumour tissue samples among 63 patients. (A) Genomic profiling of some high-frequency mutations between baseline tissue samples and plasma samples (nonsynonymous single-nucleotide variants and indels). The top bar represents the number of mutations a patient carried; the side bar represents the number of patients who carried a certain mutation. (B) Correlation analysis between mutation frequencies of 378 genes from the NGS panel in circulating tumour DNA (ctDNA) samples versus tissue samples (Spearman's rank correlation). (C) Comparison of RAS and BRAF ${ }^{\mathrm{V} 600 \mathrm{E}}$ mutations in tissue samples and plasma samples. MUT, mutant; $\mathrm{P}$, plasma; T, tissue; WT, wild type.

correlation between the site of metastasis and baseline ctDNA levels was observed (online supplemental figure S1). The median maximum variant allele frequency (VAF) (maximum somatic allele frequency (MSAF)) was significantly higher in patients who had only liver (29.6\%; IQR, $12.4 \%-48.1 \%)$ or lymph node (41.6\%; IQR, 20.3\%-56.1\%) metastasis site, compared with those who had only lung $(1.2 \%$; IQR, $0 \%-4.7 \%)$ or non-liverlung (1.1\%; IQR, 0.7\%-2.1\%) metastasis site.

\section{Mutational concordance in paired baseline plasma and tissue} Among 63 patients with paired baseline plasma and tissue samples, we compared the consistency of somatic variants (nonsynonymous single-nucleotide variants (SNVs) and indels) between ctDNA samples (detected by NGS) and corresponding tissue samples (whole-exome sequencing, WES) (figure 2A; online supplemental table S2). Further analysis indicated that the prevalence of SNVs/indels among the 378 tumor-related genes (online supplemental table S3) included in the NGS panel of ctDNA was positively correlated with that observed in tumour tissues at baseline $\left(\mathrm{R}^{2}=0.91 ; \mathrm{p}<0.001\right.$; figure $\left.2 \mathrm{~B}\right)$. The RAS mutation rate was $46.0 \%$ in tumour tissue $(n=29)$ and $47.6 \%$ in ctDNA $(\mathrm{n}=30)$, and the $\mathrm{BRAF}^{\mathrm{V} 600 \mathrm{E}}$ mutation rate was $9.5 \%$ in tumour tissue $(n=6)$ and $7.9 \%$ in ctDNA $(n=5)$. Hence, the overall agreement of RAS/BRAF ${ }^{\mathrm{V} 600 \mathrm{E}}$ status between plasma and tissue was $81.0 \%(51 / 63)$, with $17.5 \%(11 / 63)$ and $1.5 \%$ $(1 / 63)$ disagreement in RAS and $\mathrm{BRAF}^{\mathrm{V} 600 \mathrm{E}}$ status, respectively 
(figure 2C). Furthermore, among the patients with only liver metastasis, RAS concordance rate was $90.0 \%$ (18/20). And the RAS concordance rate was $79.1 \%(34 / 43)$ in the patients with extrahepatic lesions (online supplemental figure S2). These data displayed favourable gene-level concordance between tumour tissue samples and ctDNA samples but showed some discordance in RAS and $\mathrm{BRAF}^{\mathrm{V} 600 \mathrm{E}}$ mutations.

\section{Genomic evolution in plasma ctDNA under first-line treatment}

To investigate genomic evolution under first-line treatment, we collected serial plasma samples and referred to the Oncology Knowledge Base (OncoKB), which offers evidence-based therapeutic implications of cancer alterations, and generated a picture of actionable targets in CRC (figure 3A). Among the cohort of 145 patients with sequential plasma samples before progressive disease (PD), the median time from baseline ctDNA collection to the last liquid biopsy sample collection before PD was 4.67 months (range, 1.40-14.50 months; figure 3B).

A comprehensive comparison of the top mutant genes and CRC-related actionable variants between baseline and last liquid biopsy before PD showed the overall decline in mutation frequencies in most genes after a period of treatment (figure $3 \mathrm{C}$ ). For standard-of-care (SOC) targets, 42.6\% (26/61) of patients with RAS mutations showed RAS mutation clearance and 5 of them $(5 / 61,8.2 \%)$ showed RAS clearance with detectable ctDNA. $50.0 \%(5 / 10)$ of patients with BRAF mutations showed BRAF mutation clearance, while 3.6\% (3/84) and 0.7\% (1/135) of patients showed new RAS or BRAF mutations, respectively (figure 3D). Of note, the alterations in RAS status during firstline treatment were fairly stable in our cohort (online supplemental figure S3), suggesting that the dynamic genetic change was a steady event for individual patients. In addition, one of three patients lost ERBB2 amplification after treatment, while none of the patients acquired new ERBB2 amplification. Unlike SOC targets, actionable variants for clinical trials, including $\mathrm{KRAS}^{\mathrm{G} 12 \mathrm{C}}$, $\mathrm{BRAF}^{\mathrm{nonV600E}}$, PTEN, NF1, MTOR, CDK12, CDKN2A, FGFR1/FGFR2/FGFR3 alterations, changed in $12.4 \%(18 / 145)$ of patients but remained consistent over time in most $(127 / 145,87.6 \%)$ patients (figure 3E). No NTRK fusions were detected at baseline or after treatment.

\section{Clinical outcomes according to shift in plasma RAS and BRAF $^{\mathrm{V} 600 \mathrm{E}}$}

To further evaluate the importance of RAS and BRAF mutations in reflecting prognosis, we analysed the association between the dynamic changes in RAS and BRAF status shown in figure 3D and clinical outcomes.

Of note, in our cohort, ctDNA levels were positively correlated with tumour burden during first-line treatment (online supplemental figure S4A, B). Furthermore, the median ctDNA level was $14.0 \%$ (IQR, 2.0\%-47.4\%) at baseline, which was significantly higher than that of $0.5 \%$ (IQR, 0\%-4.0\%) at last biopsies before PD among 145 patients $(\mathrm{p}<0.001$, online supplemental figure S4C), revealing a significant decline in ctDNA levels after a period of treatment. So, changes in ctDNA levels were adjusted when survival analyses were performed.

The median progression-free survival (mPFS) of patients with RAS or BRAF clearance were 12.8 months (95\% CI 10.2 to not reached (NR)) and NR (95\% CI 18.9 to NR), respectively, which were similar to the mPFS of 13.2 months (95\% CI 10.8 to 16.4 ; $\mathrm{p}=0.980)$ and 11.5 months $(95 \%$ CI 10.4 to $13.3 ; \mathrm{p}=0.196)$ in patients who remained free of RAS or BRAF mutation but were significantly better than the MPFS of patients who remained RAS mutant $(7.9$ months; $95 \%$ CI 5.1 to $10.9 ; \mathrm{p}=0.002)$ or BRAF mutant (6.4 months; $95 \%$ CI 3.2 to $\mathrm{NR}$; $\mathrm{p}=0.002$; figure $4 \mathrm{~A}$, table 1). Similarly, patients with RAS or BRAF clearance showed a similar median overall survival (mOS) versus patients who remained RAS wild-type (NR (95\% CI 22.7 to NR) versus 31.4 (95\% CI 26.0 to NR); $\mathrm{p}=0.906$ ) or BRAF wild-type (NR (95\% CI 20.8 to NR) versus 26.7 months (95\% CI 22.7 to NR); $\mathrm{p}=0.869)$, whereas patients who maintained RAS or BRAF mutations had a shorter mOS of 14.5 months (95\% CI 12.7 to $\mathrm{NR} ; \mathrm{p}=0.006)$ and 11.4 months $(95 \% \mathrm{CI} 5.6$ to $\mathrm{NR} ; \mathrm{p}=0.022)$, respectively (figure $4 \mathrm{~B}$, table 1 ).

In contrast, the mPFS in patients with new RAS or BRAF mutations was 6.1 months (95\% CI 2.2 to NR) and 8.7 months (95\% CI NR to NR), respectively, which were similar to the mPFS of patients who maintained RAS or BRAF mutations (7.9 months, $\mathrm{p}=0.350 ; 6.4$ months, $\mathrm{p}=0.774$ ) and were numerically shorter than the mPFS of patients who remained RAS or BRAF wild-type (13.2 months, $\mathrm{p}=0.014 ; 11.5$ months, $\mathrm{p}=0.179$; figure $4 \mathrm{~A}$, table 1). There were no differences in the mOS of patients with RAS or BRAF acquisition (15.0 months $(95 \% \mathrm{CI}$ 11.3 to NR); NR (95\% CI NR to NR)) compared with those who remained RAS or BRAF mutations (14.5 months, $p=0.262$; 11.4 months, $\mathrm{p}=0.996)$. However, patients who acquired RAS mutations showed a shorter mOS than those who remained RAS wild-type (31.4 months, $\mathrm{p}=0.003$; figure $4 \mathrm{~B}$ ).

Genomic evolution in plasma ctDNA after disease progression Faced with second-line clinical decisions, we tracked the clinical variant dynamics of mCRC in 20 patients from whom plasma samples were obtained after PD (median time interval since baseline, 6.57 months; range, 1.07-12.20 months) following first-line therapies (figure 5A). For SOC targets, 44.4\% (4/9) of patients with RAS mutations showed RAS clearance, and none of the patients showed BRAF clearance, while 27.3\% (3/11) and $5.3 \%(1 / 19)$ of patients showed new RAS or BRAF mutations, respectively. ERBB2 remained wild-type in all 20 patients after PD (figure 5B). Moreover, for clinical-trial variants, only 1 patient obtained a new NF1 mutation, whereas 95.0\% (19/20) of patients exhibited no changes over time (figure $5 \mathrm{C}$ ).

Meanwhile, ctDNA levels at best response (median MSAF, $0.1 \%$; IQR, 0\%-1.0\%) during treatment were significantly lower than that at the baseline (median MSAF, 8.5\%; IQR, $2.7 \%-55.9 \% ; \mathrm{p}<0.001$ ) and the disease progression (median MSAF, 10.4\%; IQR, 0.7\%-27.9\%; $\mathrm{p}=0.002$ ). The ctDNA levels decreased in patients with partial response or stable disease and increased when PD was observed, allowing the use of ctDNA detection in response evaluation (online supplemental figure S5).

Furthermore, to identify potential mechanisms of resistance to EGFR antibody (EGFR-Ab) treatment, we analysed the genetic changes between baseline and PD plasma samples of six patients who received EGFR-Abs as a first-line regimen (online supplemental figure S6). Compared with baseline samples, PD samples from four patients exhibited the most peculiar genetic alterations in genes involved in the RTK-RAS pathway and PI3K pathway, including mutations of RAS, BRAF, ALK, HGF, NF1, and PIK3CG and amplification of MET. We also detected the acquisition of several genetic alterations that likely confer EGFR-Ab resistance, as described in recent studies, ${ }^{25-28}$ such as mutations of ATM, SMAD2 and CCNE1 amplification. GATA2, a necessary transcription factor for RAS-mutant non-small-cell lung cancer cells, ${ }^{29}$ was detected in one patient at PD. New mutations in SYNE1, IKZF1 and TERT amplification were observed 


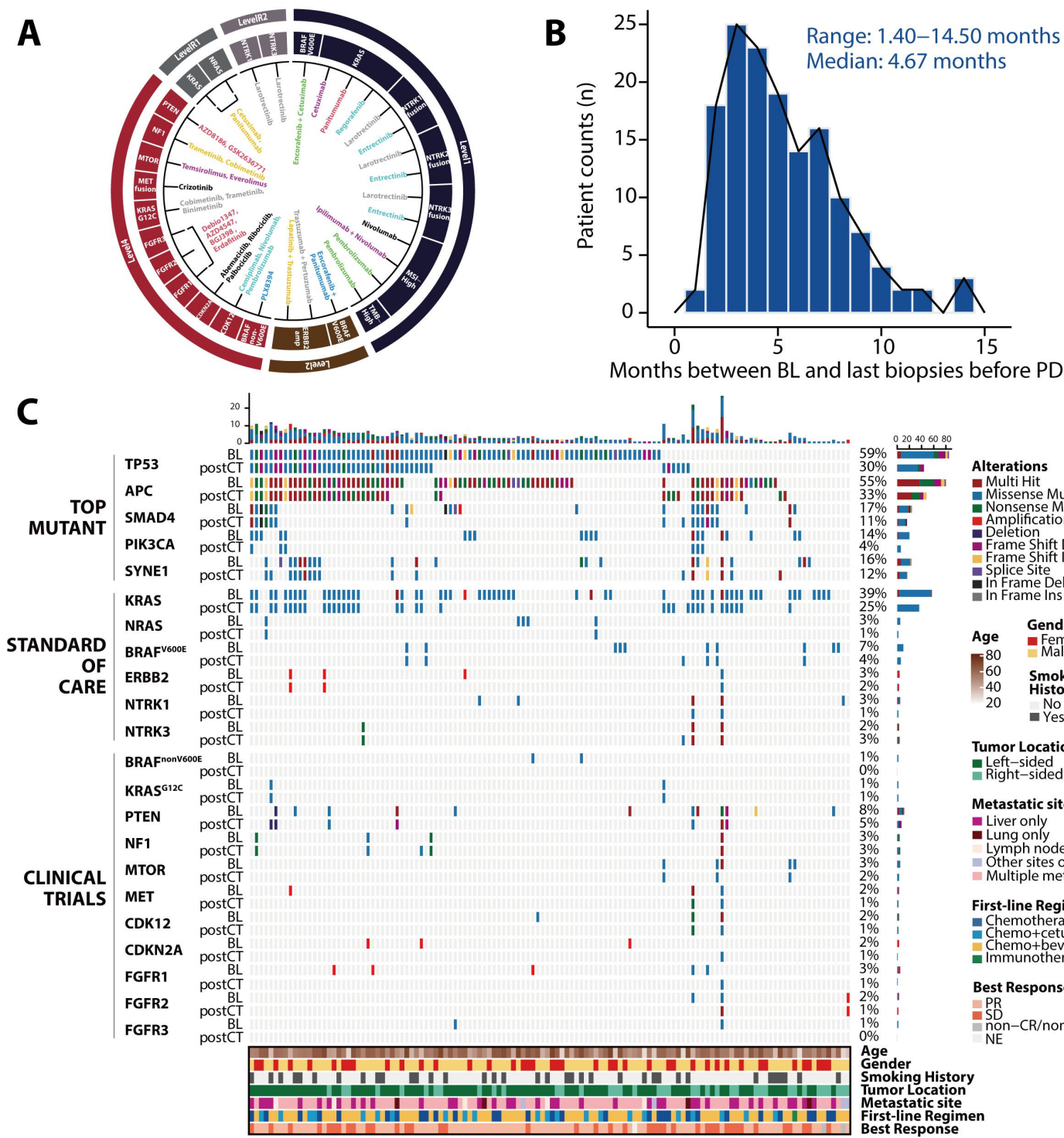

Months between $\mathrm{BL}$ and last biopsies before PD

Figure 3 Genomic temporal heterogeneity in plasma circulating tumour DNA under first-line treatment among 145 patients. (A) Evidence-based actionable targets in colorectal cancer (CRC) referring to the Oncology Knowledge Base database. The outer ring represents the different levels of evidence for the actionable targets, and the inner part represents the different treatments according to the corresponding targets. (B) Time from baseline plasma sample collection to the last liquid biopsy before progressive disease (PD). (C) Genomic profiling of the most commonly mutated genes and actionable targets in CRC between baseline and post-chemotherapy (postCT) plasma samples. The top bar represents the number of mutations a patient carried; the side bar represents the number of patients who carried a certain mutation; and the bottom bar represents patient characteristics, including age, sex, smoking history, tumour location, metastatic site at baseline, best response and first-line chemotherapy regimen. (D) The clearance and acquisition rates of standard-of-care targets and top mutant genes after treatment. (E) The shift of actionable targets for clinical trials after treatment. amp, amplification; $\mathrm{BL}$, baseline; $\mathrm{CR}$, complete response; MSI, microsatellite instability; NE, not evaluable; PR, partial response; SD, stable disease; TMB, tumour mutation burden. 

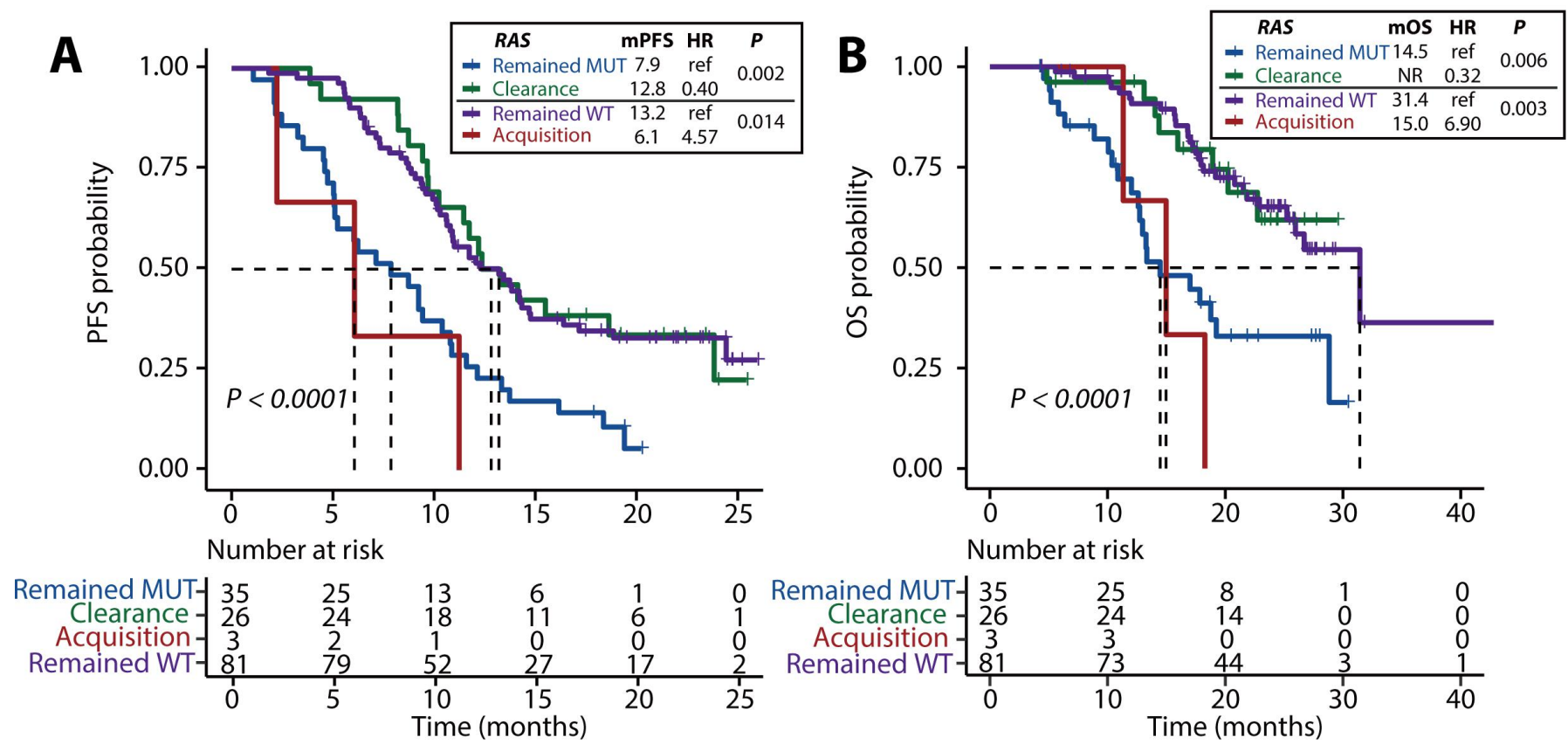

Figure 4 Kaplan-Meier estimates of progression-free survival (PFS) (A) and overall survival (OS) (B) in patients stratified according to different changes in plasma RAS status under first-line treatment Statistical significance was determined by Wald test of the multivariable Cox models. The change in the circulating tumour DNA (ctDNA) fraction of cfDNA, estimated by maximum somatic allele frequency, was included as a variable. mPFS, median progression-free survival; mOS, median overall survival; MUT, mutant; ref, reference; WT, wild-type.

in patients who showed resistance to EGFR-Abs. Collectively, these results indicate that the mechanisms of anti-EGFR drug resistance might be complex and probably cannot be explained by the presence of a single genetic alteration. A larger cohort of patients is needed to verify these potential mechanisms.

\section{DISCUSSION}

In the current study, we observed good concordance of somatic mutations detected by tumour tissue samples and matched plasma ctDNA samples, showing the reliability ctDNA in the detection of somatic variants during systemic therapy for mCRC. Strickler et al showed that ctDNA profiling of CRC can be used to detect somatic mutations at frequencies comparable to those observed by tumour sample sequencing in other independent cohorts. ${ }^{15}$ In this study, we provide more solid evidence to show that ctDNA profiling can be used as an alternative to tissue profiling and can accurately capture the somatic mutational spectrum of primary tumours. These results indicate that for patients with mCRC, ctDNA profiling could be a reliable option to gain insight into the somatic mutational landscape to guide treatment decisionmaking. Furthermore, repeated tissue biopsy for genomic profiling is impractical. Thus, subsequent treatment decisionmaking must rely on archival tumour tissues, which are unable to reflect the dynamic evolution of the primary tumour and metastatic lesions. ${ }^{30-32}$ The good concordance of tumour tissue sequencing and ctDNA profiling also indicates that the latter is reliable and can be used in dynamic and real-time testing during the clinical course of disease, especially when the patients face treatment failure and require further treatment.

Based on serial ctDNA profiling, we thoroughly analysed the temporal heterogeneity of CRC-related somatic variants under first-line systemic therapy until PD, depicting the landscape of somatic variant shifts, and their association with treatment outcomes and implications for clinical practice. Notably, during first-line therapy, a significant shift in the somatic mutation status of recurrently mutated genes in CRC was observed, supporting that dynamic and real-time ctDNA profiling are needed to capture the real somatic mutational spectrum when making treatment decisions, which would benefit patients more than a static view of this spectrum. ${ }^{11} 12$

Recently, clearance of plasma RAS mutations in mCRC was used as a biomarker for selecting patients eligible for anti-EGFR

\begin{tabular}{|c|c|c|c|c|c|c|c|c|c|}
\hline$B R A F^{V 600 E}$ & No. & Events & $\begin{array}{l}\text { mPFS, } 95 \% \mathrm{Cl} \\
\text { (months) }\end{array}$ & HR & $P^{*}$ & Events & $\begin{array}{l}\text { mOS, } 95 \% \mathrm{Cl} \\
\text { (months) }\end{array}$ & HR & $P^{*}$ \\
\hline $\begin{array}{l}\text { Remained } \\
\text { MUT }\end{array}$ & 5 & 5 & $6.4,3.2$ to $N R$ & ref & 0.002 & 3 & $11.4,5.6$ to NR & ref & 0.022 \\
\hline Clearance & 5 & 2 & $\mathrm{NR}, 18.9$ to NR & 0.07 & & 2 & $N R, 20.8$ to NR & 0.09 & \\
\hline $\begin{array}{l}\text { Remained } \\
\text { WT }\end{array}$ & 134 & 97 & $11.5,10.4$ to 13.3 & ref & 0.179 & 55 & $26.7,22.7$ to $N R$ & ref & 0.996 \\
\hline Acquisition & 1 & 1 & $8.7, N R$ to $N R$ & 3.95 & & 0 & $N R$, NR to NR & $<0.01$ & \\
\hline
\end{tabular}

\footnotetext{
*Statistical significance was determined by Wald test of the multivariable Cox models. The change in the ctDNA fraction of cfDNA, estimated by MSAF, was included as a variable.
} mOS, median overall survival; mPFS, median progression-free survival; MUT, mutant; NR, not reached; ref, reference; WT, wild-type. 


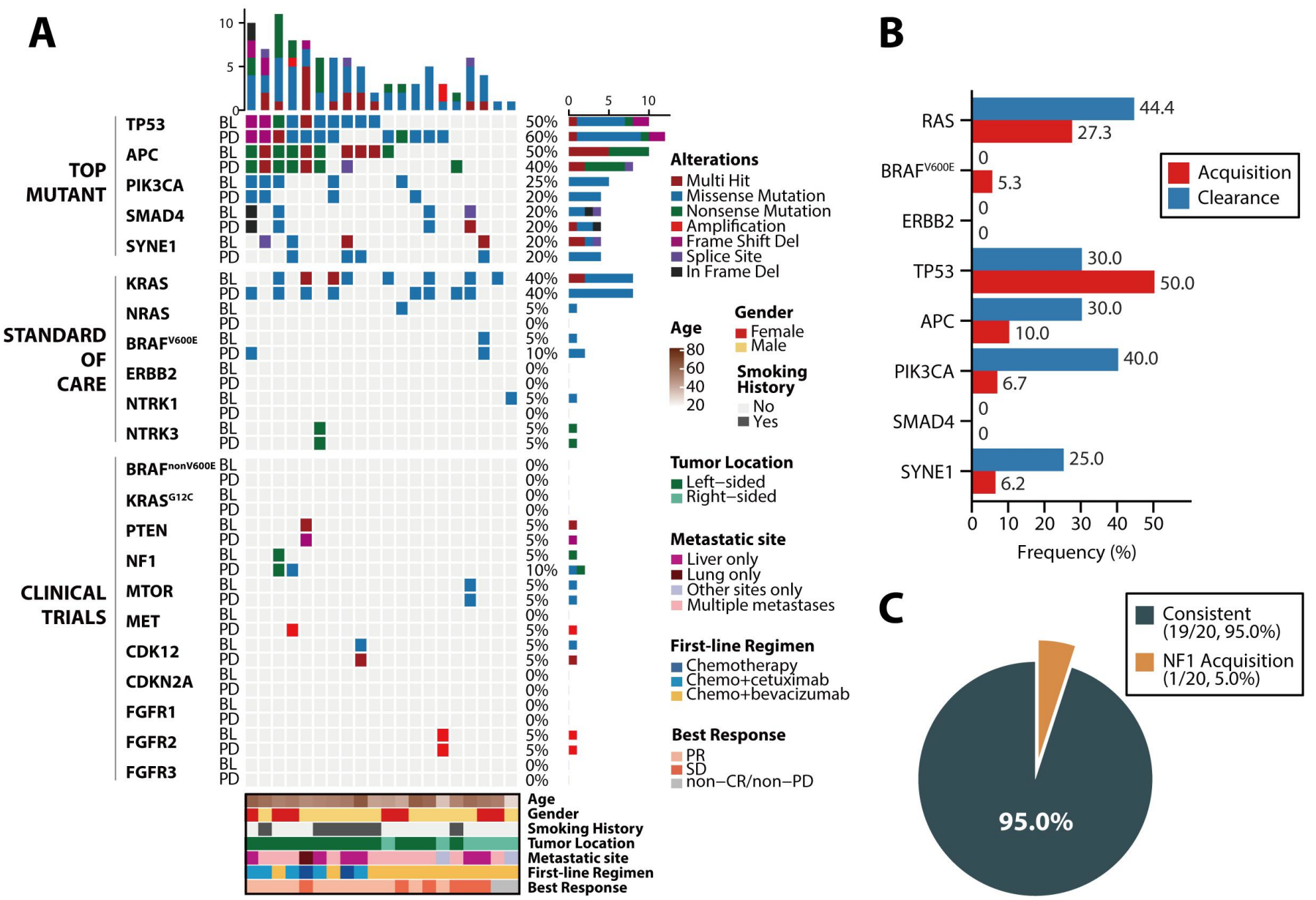

Figure 5 Genomic temporal heterogeneity under a period of first-line regimen after progressive disease among 20 patients. (A) Genomic profiling of the top mutant genes and actionable targets in colorectal cancer between baseline and progressive disease plasma samples. The top bar represents the number of mutations a patient carried; the side bar represents the number of patients who carried a certain mutation; and the bottom bar represents patient characteristics, including age, sex, smoking history, tumour location, metastatic site at baseline, best response and first-line chemotherapy regimen. (B) The prevalence of clearance and acquisition of standard-of-care targets and the top mutant genes after progression. (C) The shift of actionable targets for clinical trials after progression. $C R$, complete response; PD, progressive disease; PR, partial response; SD, stable disease.

rechallenge, and this change occurs at a highly variable rate ranging from $2 \%$ to $45 \% .^{33-37}$ However, RAS reversion in mCRC before disease progression has rarely been investigated. In this study, we paid close attention to the reversion of genetic mutation status in ctDNA before PD, and our data showed a relatively high rate of RAS clearance before PD $(42.6 \%, 26 / 61)$. Notably, taking presence of ctDNA into consideration, $34.4 \%$ of patients had complete ctDNA clearance and only $8.2 \%$ of patients showed RAS clearance with detectable ctDNA. In addition, the stability of the shift of RAS mutational status during treatment was observed by serial ctDNA testing (online supplemental figure S3); the evidence supported the existence of positive or negative selection of RAS-mutated clones rather than technical errors. In this aspect, more data are warranted to reflect the effects of RAS clearance, along with complete ctDNA clearance or not, on the future therapeutic implication, such as anti-EGFR rechallenge.

The most interesting and clinically relevant finding was that the shift of somatic mutational status of plasma RAS or BRAF genes was accompanied by a drastic change in clinical outcomes, with improved efficacy and survival in patients whose phenotype shifted from RAS/BRAF-mutant to wild-type. These observations indicated that the initial somatic mutational status may not always be reliable in prognostic stratification and treatment decision-making. Instead, subsequent and real-time mutational status may have greater impacts on treatment efficacy and patient survival. Nevertheless, the change in ctDNA fraction in cell-free DNA (cfDNA) was taken into consideration by calculating the MSAF when we investigated the impact of a shift in RAS/BRAF status on prognosis, which further supported the strong correlations between plasma RAS/BRAF dynamics and clinical outcomes.

Likewise, we observed significant changes in the somatic mutational landscape at the time of treatment failure of first-line systemic therapy. This could partially explain the reason for treatment failure and provide us with more information to determine the subsequent treatments. For example, in six patients treated with anti-EGFR therapy, new somatic alterations emerged after the failure of anti-EGFR therapy. Most of these alterations were involved in RTK-RAS and PI3K pathway, which could be the potential resistance mechanisms to EGFR therapy. However, more detailed verifications of these alterations are expected to reveal the acquired resistance mechanisms to EGFR therapy. Besides, changes in clinical targets, especially RAS and BRAF, also have implications for decision-making regarding subsequent lines of treatment. These results emphasise the importance of 
dynamic monitoring using ctDNA profiling, especially at the time of treatment failure, for determining the best treatment options.

Several limitations should be acknowledged in this study. First, the ctDNA/tumour RAS discordance rate was $17.5 \%$ at baseline. The most plausible cause of this discordance is that somatic mutations were not detected in baseline plasma samples of a small proportion of patients (16.4\%), which is in consist with previous studies. ${ }^{15} 3839$ The median MSAF of the RASconcordant subgroup was significantly higher than that of the RAS-discordant subgroup (27.3\% vs $1.5 \%)$, which supported this speculation to some extent. Besides, the ctDNA/tumour discordance level was reported to differ by metastatic sites. ${ }^{40}$ In our cohort, RAS concordance rate was $90.0 \%$ among patients with only liver metastasis, while the rate of patients with extrahepatic lesions was down to $79.1 \%$. To increase the sensitivity for mutation detection in the ctDNA, further technical improvement in mutation detection is needed. Second, as the somatic mutational rate of BRAF is rather low, the actual number of patients who present with a shift in somatic mutational status is also not sufficient for statistical testing. Thus, the prognostic impact of the shift in BRAF somatic mutational status still warrants further confirmation, as well as its predictive implication for BRAF inhibitors. Third, although the PFS data are mature in the study, an extended long-term follow-up is needed to confirm the OS findings.

In conclusion, this prospective, observational and large-scale ctDNA profiling study provided further and solid evidence to support the use of ctDNA sequencing in capturing the dynamic somatic mutational spectrum of mCRCs. More importantly, we revealed the temporal heterogeneity of mCRC-related somatic variants by serial ctDNA profiling, which should be given special attention in clinical practice, as evidenced by the finding that the shift in plasma somatic mutational status of the RAS or BRAF genes was accompanied by a drastic change in survival outcomes.

\section{MATERIALS AND METHODS}

\section{Patients and samples collection}

A prospective cohort study (ClinicalTrials.gov identifier: NCT04228614) was designed and implemented in the Sun Yatsen University Cancer Center (Guangzhou, China). A total of 171 patients with unresectable mCRC under first-line treatment between April 2018 and January 2020 were enrolled. Patient blood samples with or without primary tumour samples were sequentially collected at baseline and every 6-8 weeks with response evaluation until PD or last follow-up. Clinical response and tumour burden were evaluated by the investigators according to the Response Evaluation Criteria in Solid Tumours, V.1.1. The inclusion criteria for the patients were as follows: (1) the patients were diagnosed with mCRC by histopathology or cytology by qualified pathologists from Sun Yat-sen University Cancer Center; (2) the patient who had not received first-line treatment before baseline sample collection; and (3) the patients who had qualified baseline and serial plasma samples (at least $20 \mathrm{ng}$ DNA yielded) or patients who had only baseline plasma samples but had paired qualified primary tumour tissue (at least 50 ng DNA yielded). The patients who received curative resection or ablation of the metastatic lesions during the first-line treatment and achieved no evidence of disease were excluded from the final analysis. All patients consented to an institutional review boardapproved protocol for prospective tumour genomic profiling. This study was approved by the Institutional Review Board of Sun Yat-sen University Cancer Center and complied with the ethical standards of the institutional and/or national research committee and with the Declaration of Helsinki.

\section{Patient and public involvement}

It was not appropriate or possible to involve patients or the public in the design, or conduct, or reporting, or dissemination plans of our research.

DNA extraction, library construction and targeted sequencing The details of DNA extraction, library construction and targeted sequencing, including NGS of cfDNA and WES of tissue tumour DNA, were described in the online supplemental methods.

\section{Raw data processing and alignment}

Raw sequencing data were preprocessed by fastp V.0.18.0; preprocessing included adaptor trimming, removal of the reads in which the $\mathrm{N}$ base reached a certain percentage (default length of $5 \mathrm{bp}$ ) and reads that contained low-quality bases (default quality threshold value $\leq 20$ ) above a certain portion (default $40 \%$ ), and sliding window trimming. ${ }^{41}$ Clean reads were aligned to the hg19 genome (GRCh37) using Burrows-Wheeler Aligner V.0.7.15-r1140 with the default settings. ${ }^{42}$ GenCore V.0.12.0 was used to remove duplicate reads. ${ }^{43}$ Samtools V.0.1.19 was applied to generate pileup files for properly paired reads with mapping quality $\geq 60$. $^{44}$

\section{Mutation calling, filtering and annotation}

After removing duplicate reads, the mean coverage depth was $1000 \times$ for the whole blood control samples, $250 \times$ for tumour tissues and $2000 \times$ for cfDNA samples. For ctDNA-NGS, SNVs and short insertions/deletions (indels) were identified by VarScan2 V.2.3.8; the minimum read depth was 200, and the VAF threshold was set to $0.1 \%{ }^{45}$ Somatic variants (SNVs or indels) presenting at least five unique reads, with at least one on each strand, and with a mutant allelic frequency less than $0.5 \%$ in the paired normal sample (peripheral blood lymphocytes) were retained. Additionally, we excluded any SNVs by background polishing using cfDNA samples from healthy subjects (online supplemental methods). A manual visual inspection step was applied to further remove artefacts by GenomeBrowse (http://www.goldenhelix.com). For tissue WES, somatic variants identified by at least two out of the three callers (VarScan $2,{ }^{45} \mathrm{TNscope}^{46}$ and Mutect $2^{47}$ ) were selected and then filtered with three criteria: (1) VAF $\geq 8 \%$; (2) sequencing depth in the region $\geq 8$; and (3) sequence reads in support of the variant call $\geq 2$. All SNVs/indels were annotated using ANNOVAR (Annotate Variation, V.201804-16). ${ }^{48}$ CNVkit V.0.9.3 was used for copy number variation (CNV) detection of the ctDNA samples ${ }^{49}$; EXCAVATOR2 V.1.1.2 was used for CNV analysis, ${ }^{50}$ and GeneFuse V.0.6.1 was applied for structural variation detection. ${ }^{51}$

\section{Selection of genomic alterations}

Somatic variants (SNVs or indels) were included for the comparison of mutational concordance between tumour tissue and plasma ctDNA. To explore genomic evolution under treatment in CRC, gene alterations were filtered for oncogenic variants using the OncoKB, a comprehensive and curated database that offers detailed, evidence-based information about individual somatic mutations and structural alterations with potential clinical actionability that are present in patient tumours. ${ }^{52}$ Targets with level one or two evidence, including KRAS, NRAS and $\mathrm{BRAF}^{\mathrm{V} 600 \mathrm{E}}$ mutations as well as NTRK fusion and ERBB2 amplification, were defined as SOC actionable variants. Targets with 
level four evidence, including KRAS ${ }^{\mathrm{G} 12 \mathrm{C}}$, BRAF $^{\mathrm{nonV600E}}$ (G464, G469A, G469R, G469V, K601, L597), PTEN, NF1, MTOR, CDK12, CDKN2A, and FGFR1/FGFR2/FGFR3 alterations as well as MET fusion, were adopted as variants for clinical trials (figure 3A). To investigate the potential resistance mechanisms of EGFR antibodies, all new genetic alterations were noted.

\section{Statistical analysis}

The analysis of concordance between plasma and primary tumour mutational status for each gene was based on overall percent agreement, sensitivity (positive percent agreement), and specificity (negative percent agreement). The MSAF was calculated for each case and used to provide an estimate of the ctDNA fraction in the blood. ${ }^{53}$ PFS was defined as the time from enrollment to disease progression, death, or the end of follow-up, whichever came first. OS time was measured from the date of diagnosis of stage IV disease until the date of death or last follow-up. For survival tests, PFS and OS were analysed using the KaplanMeier method. For comparison of PFS or OS between different groups, the log-rank test was used. A multivariable Cox proportional hazards model was established to adjust for the impact of changes in the ctDNA fraction of cfDNA. Correlations between variables were assessed using Spearman's rank correlation coefficient. A two-tailed $\mathrm{p}<0.05$ was considered to be statistically significant. Statistical analysis was performed with R (V.4.0.1).

\section{Author affiliations \\ 'Department of Medical Oncology, Sun Yat-sen University Cancer Center, State Key Laboratory of Oncology in South China, Collaborative Innovation Center for Cancer Medicine, Sun Yat-sen University, Guangzhou, Guangdong, People's Republic of China \\ ${ }^{2}$ Research Unit of Precision Diagnosis and Treatment for Gastrointestinal Cancer, Chinese Academy of Medical Sciences, Guangzhou, Guangdong, People's Republic of China \\ ${ }^{3}$ Bioinformatics Platform, Sun Yat-sen University Cancer Center, Guangzhou, Guangdong, People's Republic of China \\ ${ }^{4}$ Research and Development Division, HaploX Biotechnology, Shenzhen, Guangdong, People's Republic of China}

Acknowledgements We thank all patients and their families for participating in this study.

Contributors Conception and design: FW, R-HX. Development of methodology: Y-SH, H-XW, Z-XW, YJ, Y-XC, M-MH, S-fC, M-yX, QZ. Acquisition of data: Y-SH, H-XW, Z-XW, YJ, Y-XC, QZ, M-MH, H-YL, M-ZQ, D-sW, F-HW, Y-HL, FW, R-HX. Analysis and interpretation of data: Y-SH, H-XW, Y-XC, S-fC, M-yX, QZ, FW, R-HX. Writing, review, and/or revision of the manuscript: Y-SH, H-XW, Z-XW, FW, R-HX. Study supervision: FW, R-HX.

Funding The National Key R\&D Program of China (2018YFC1313300, to R-HX): the National Natural Science Foundation of China (81930065, to R-HX); the International Cooperation and Exchanges National Natural Science Foundation of China (82061160373, to FW); the National Natural Science Foundation of China (General Program, 81872011, to FW); the Science and Technology Program of Guangdong (2019B020227002, to R-HX); the Science and Technology Program of Guangzhou (201904020046, 201803040019, 201704020228, to R-HX); the Sun Yat-sen University Clinical Research 5010 Program (2018014, to FW); the Young Physician Scientist Program of Sun Yat-sen University Cancer Center (16zxqk03, to FW); the Fundamental Research Funds of Sun Yat-sen University Cancer Center (20ykzd166, to YJ); the Precision Oncology Research Program of Guangdong (to YJ).

Competing interests None declared.

Patient consent for publication Not required.

Provenance and peer review Not commissioned; externally peer reviewed.

Data availability statement Data are available upon reasonable request.

Supplemental material This content has been supplied by the author(s). It has not been vetted by BMJ Publishing Group Limited (BMJ) and may not have been peer-reviewed. Any opinions or recommendations discussed are solely those of the author(s) and are not endorsed by BMJ. BMJ disclaims all liability and responsibility arising from any reliance placed on the content. Where the content includes any translated material, BMJ does not warrant the accuracy and reliability of the translations (including but not limited to local regulations, clinical guidelines, terminology, drug names and drug dosages), and is not responsible for any error and/or omissions arising from translation and adaptation or otherwise.

Open access This is an open access article distributed in accordance with the Creative Commons Attribution Non Commercial (CC BY-NC 4.0) license, which permits others to distribute, remix, adapt, build upon this work non-commercially, and license their derivative works on different terms, provided the original work is properly cited, appropriate credit is given, any changes made indicated, and the use is non-commercial. See: http://creativecommons.org/licenses/by-nc/4.0/.

ORCID iD

Rui-Hua Xu http://orcid.org/0000-0001-9771-8534

\section{REFERENCES}

1 Siegel RL, Miller KD, Goding Sauer A, et al. Colorectal cancer statistics, 2020. CA Cancer J Clin 2020;70:145-64.

2 Siegel RL, Miller KD, Jemal A. Cancer statistics, 2020. CA Cancer J Clin 2020;70:7-30.

3 Dienstmann R, Vermeulen L, Guinney J, et al. Consensus molecular subtypes and the evolution of precision medicine in colorectal cancer. Nat Rev Cancer 2017;17:79-92.

4 Wang F, Wang Z-X, Chen G, et al. Expert opinions on immunotherapy for patients with colorectal cancer. Cancer Commun 2020;40:467-72.

5 Wang Z-X, Wu H-X, He M-M, et al. Chemotherapy with or without anti-EGFR agents in left- and right-sided metastatic colorectal cancer: an updated meta-analysis. J Nat/ Compr Canc Netw 2019;17:805-11.

6 Dienstmann R, Ciner A, Hochster HS. Should next-generation sequencing testing be routinely used in metastatic colorectal cancer? Lancet Oncol 2018;19:1434-5.

7 Innocenti F, Ou F-S, Qu X, et al. Mutational analysis of patients with colorectal cancer in CALGB/SWOG 80405 identifies new roles of microsatellite instability and tumor mutational burden for patient outcome. J Clin Oncol 2019;37:1217-27.

8 Kopetz S, Grothey A, Yaeger R, et al. Encorafenib, binimetinib, and cetuximab in BRAF V600E-mutated colorectal cancer. N Engl I Med 2019;381:1632-43.

9 Misale S, Di Nicolantonio F, Sartore-Bianchi A, et al. Resistance to anti-EGFR therapy in colorectal cancer: from heterogeneity to convergent evolution. Cancer Discov 2014:4:1269-80.

10 Wang Z-X, Yao Y-C, Mai Z-J. Temporal change in treatment patterns of metastatic colorectal cancer and its association with patient survival: a retrospective cohort study based on an intelligent Big-Data platform. Engineering 2021.

11 Dagogo-Jack I, Shaw AT. Tumour heterogeneity and resistance to cancer therapies. Nat Rev Clin Oncol 2018;15:81-94

12 Heitzer E, Haque IS, Roberts CES, et al. Current and future perspectives of liquid biopsies in genomics-driven oncology. Nat Rev Genet 2019;20:71-88.

13 Xu R-H, Wei W, Krawczyk M, et al. Circulating tumour DNA methylation markers for diagnosis and prognosis of hepatocellular carcinoma. Nat Mater 2017;16:1155-61.

14 Dasari A, Morris VK, Allegra CJ, et al. ctDNA applications and integration in colorectal cancer: an NCl colon and Rectal-Anal task forces whitepaper. Nat Rev Clin Oncol 2020;17:757-70.

15 Strickler JH, Loree JM, Ahronian LG, et al. Genomic landscape of cell-free DNA in patients with colorectal cancer. Cancer Discov 2018;8:164-73.

16 Ignatiadis M, Sledge GW, Jeffrey SS. Liquid biopsy enters the clinic - implementation issues and future challenges. Nat Rev Clin Oncol 2021;18:297-312.

17 Wang $\mathrm{Y}$, Wang $\mathrm{M}, \mathrm{Wu} \mathrm{H}-\mathrm{X}$, et al. Advancing to the era of cancer immunotherapy. Cancer Commun 2021. doi:10.1002/cac2.12178. [Epub ahead of print: 24 Jun 2021].

18 Luo H, Zhao Q, Wei W, et al. Circulating tumor DNA methylation profiles enable early diagnosis, prognosis prediction, and screening for colorectal cancer. Sci Trans/ Med 2020;12:eaax7533.

19 Reinert T, Henriksen TV, Christensen E, et al. Analysis of plasma cell-free DNA by ultradeep sequencing in patients with stages I to III colorectal cancer. JAMA Oncol 2019:5:1124.

20 Tie J, Cohen JD, Wang Y, et al. Serial circulating tumour DNA analysis during multimodality treatment of locally advanced rectal cancer: a prospective biomarker study. Gut 2019;68:663-71.

21 Chen G, Peng J, Xiao Q, et al. Postoperative circulating tumor DNA as markers of recurrence risk in stages II to III colorectal cancer. J Hematol Oncol 2021;14:80.

22 Osumi H, Shinozaki E, Yamaguchi K, et al. Early change in circulating tumor DNA as a potential predictor of response to chemotherapy in patients with metastatic colorectal cancer. Sci Rep 2019;9:17358.

23 Tie J, Kinde I, Wang Y, et al. Circulating tumor DNA as an early marker of therapeutic response in patients with metastatic colorectal cancer. Ann Oncol 2015;26:1715-22.

24 Siravegna G, Mussolin B, Buscarino M, et al. Clonal evolution and resistance to EGFR blockade in the blood of colorectal cancer patients. Nat Med 2015;21:795-801.

25 Martinelli E, Ciardiello D, Martini G, et al. Implementing anti-epidermal growth factor receptor (EGFR) therapy in metastatic colorectal cancer: challenges and future perspectives. Ann Oncol 2020;31:30-40.

26 Porru M, Pompili L, Caruso C, et al. Targeting KRAS in metastatic colorectal cancer: current strategies and emerging opportunities. J Exp Clin Cancer Res 2018;37:57.

27 Bray SM, Lee J, Kim ST, et al. Genomic characterization of intrinsic and acquired resistance to cetuximab in colorectal cancer patients. Sci Rep 2019;9:15365. 
28 Maron SB, Alpert L, Kwak HA, et al. Targeted therapies for targeted populations: anti-EGFR treatment for EGFR-amplified gastroesophageal adenocarcinoma. Cancer Discov 2018;8:696-713.

29 Kumar MS, Hancock DC, Molina-Arcas M, et al. The GATA2 transcriptional network is requisite for RAS oncogene-driven non-small cell lung cancer. Cell 2012;149:642-55.

30 Wan JCM, Massie C, Garcia-Corbacho J, et al. Liquid biopsies come of age: towards implementation of circulating tumour DNA. Nat Rev Cancer 2017; 17:223-38

31 De Rubis G, Rajeev Krishnan S, Bebawy M. Liquid biopsies in cancer diagnosis, monitoring, and prognosis. Trends Pharmacol Sci 2019;40:172-86.

32 Overman MJ, Modak J, Kopetz S, et al. Use of research biopsies in clinical trials: are risks and benefits adequately discussed? J Clin Oncol 2013;31:17-22.

33 Henry J, Willis J, Parseghian CM, et al. NeoRAS: incidence of RAS reversion from RAS mutated to RAS wild type. Journal of Clinical Oncology 2020;38:180-80.

34 Gazzaniga P, Raimondi C, Nicolazzo C, et al. ctDNA might expand therapeutic options for second line treatment of KRAS mutant mCRC. Annals of Oncology 2017;28:v586.

35 Moati E, Blons H, Taly V, et al. Plasma clearance of RAS mutation under therapeutic pressure is a rare event in metastatic colorectal cancer. Int J Cancer 2020;147:1185-9.

36 Fernández Montes A, Martinez Lago N, De la Cámara Gómez J, et al. Folfiri plus panitumumab as second-line treatment in mutated Ras metastatic colorectal cancer patients who converted to wild type Ras after receiving first-line FOLFOX/CAPOX plus bevacizumab-based treatment: phase II CONVERTIX trial. Annals of Oncology 2019;30:iv23-4.

37 Raimondi C, Nicolazzo C, Belardinilli F, et al. Transient disappearance of Ras mutant clones in plasma: a counterintuitive clinical use of EGFR inhibitors in Ras mutant metastatic colorectal cancer. Cancers 2019;11. doi:10.3390/cancers11010042. [Epub ahead of print: 0401 2019].

38 Morelli MP, Overman MJ, Dasari A, et al. Characterizing the patterns of clonal selection in circulating tumor DNA from patients with colorectal cancer refractory to anti-EGFR treatment. Ann Oncol 2015;26:731-6.

39 Bettegowda C, Sausen M, Leary RJ, et al. Detection of circulating tumor DNA in earlyand late-stage human malignancies. Sci Trans/ Med 2014;6:224ra24.
40 Kagawa Y, Elez E, García-Foncillas J, et al. Combined analysis of concordance between liquid and tumor tissue biopsies for RAS mutations in colorectal cancer with a single metastasis site: the METABEAM study. Clin Cancer Res 2021;27:2515-22.

41 Chen S, Zhou Y, Chen Y, et al. fastp: an ultra-fast all-in-one FASTQ preprocessor. Bioinformatics 2018;34:i884-90.

42 Li H, Durbin R. Fast and accurate long-read alignment with Burrows-Wheeler transform. Bioinformatics 2010;26:589-95.

43 Chen S, Zhou Y, Chen Y, et al. Gencore: an efficient tool to generate consensus reads for error suppressing and duplicate removing of NGS data. BMC Bioinformatics 2019;20:606.

44 Li H, Handsaker B, Wysoker A, et al. The sequence alignment/map format and SAMtools. Bioinformatics 2009;25:2078-9.

45 Koboldt DC, Zhang Q, Larson DE, et al. VarScan 2: somatic mutation and copy number alteration discovery in cancer by exome sequencing. Genome Res 2012;22:568-76.

46 Freed D, Pan R, Aldana R. TNscope: accurate detection of somatic mutations with haplotype-based variant candidate detection and machine learning filtering. bioRxiv 2018;250647.

47 Cibulskis K, Lawrence MS, Carter SL, et al. Sensitive detection of somatic point mutations in impure and heterogeneous cancer samples. Nat Biotechnol 2013;31:213-9.

48 Wang K, Li M, Hakonarson H. ANNOVAR: functional annotation of genetic variants from high-throughput sequencing data. Nucleic Acids Res 2010;38:e164.

49 Talevich E, Shain AH, Botton T, et al. CNVkit: genome-wide copy number detection and visualization from targeted DNA sequencing. PLoS Comput Biol 2016;12:e1004873.

50 D'Aurizio R, Pippucci T, Tattini L, et al. Enhanced copy number variants detection from whole-exome sequencing data using EXCAVATOR2. Nucleic Acids Res 2016;44:e154

51 Chen S, Liu M, Huang T, et al. GeneFuse: detection and visualization of target gene fusions from DNA sequencing data. Int J Biol Sci 2018;14:843-8.

52 Chakravarty D, Gao J, Phillips SM. OncoKB: a precision oncology knowledge base. JCO Precis Oncol 2017;2017. doi:10.1200/PO.17.00011. [Epub ahead of print: 1605 2017].

53 Zhou C, Yuan Z, Ma W, et al. Clinical utility of tumor genomic profiling in patients with high plasma circulating tumor DNA burden or metabolically active tumors. $J$ Hematol Oncol 2018;11:129. 